\title{
Global science discussed in local altmetrics: Weibo and its comparison with Twitter
}

\author{
Houqiang $\mathrm{Yu}^{\mathrm{a}}$, Shenmeng Xu${ }^{\mathrm{b}, *}$, Tingting Xiao ${ }^{\mathrm{a}}$, Brad M. Hemminger ${ }^{\mathrm{b}}$, \\ Siluo Yang ${ }^{\mathrm{a}}$ \\ a School of Information Management, Wuhan University, Wuhan 430072, China \\ b School of Information and Library Science, UNC Chapel Hill, Chapel Hill, 27514, USA
}

\section{A R T I C L E I N F O}

\section{Article history:}

Received 18 July 2016

Received in revised form 27 February 2017

Accepted 27 February 2017

Available online 20 March 2017

Keywords:

Altmetrics

Weibo

Altmetrics indicators

Scholarly communication

Distribution

\begin{abstract}
A B S T R A C T
Local altmetrics is currently an integral part of the altmetrics landscape. This paper aims to investigate the characteristics of microblog altmetrics of the Chinese microblog platform, Weibo, to shed light on cultural differences and draw attention to local altmetrics in developing countries. The analysis is based on 4.4 million records provided by Altmetric.com. Data collected are from March 2014 to July 2015. It is found that Weibo users discuss global science, more actively compared with several international altmetrics sources. Statistical results show strong evidence of the immediacy advantage of metrics based on Weibo as well as Twitter and the general altmetrics over citations. Distribution of Weibo altmetrics on the article level, source level and discipline level are highly skewed. Overall, compared with Twitter, Weibo altmetrics present similar distributions, with some minor variations. To better understand how and why Weibo users discuss global scientific articles, the top weiboed articles, sources and disciplines are identified and further explored. Our content analysis shows that the common motivation of scientific weibos is to disseminate or discuss the articles because they are interesting, surprising, academically useful or practically useful. Conclusion of articles is the most frequently mentioned element in scientific weibos. In addition, different from Twitter, Weibo users have a preference for traditional prestigious journals.
\end{abstract}

(C) 2017 Elsevier Ltd. All rights reserved.

\section{Introduction}

A series of behaviors are conducted in the research process, including but not limited to information seeking, saving, reading, annotating, brainstorming, experimentation, data analysis, paper drafting and citing. The citing behavior is recorded by citations, giving birth to citation analysis; in the modern digital era, many behaviors are recorded by online scholarly tools and platforms, laying the foundation of altmetrics. Altmetrics, by capturing digital traces of scientific products, aims to improve scholarly communication, scientific evaluation and literature discovery (Moed, 2015). Altmetrics research is developing from theoretical (Priem, 2013) and critical debate (Qiu \& Yu, 2015) to more empirical (Wang, Guo \& Zhang, 2015), experimental (Friedrich, Bowman, Stock, \& Haustein, 2015) and application-oriented (Das, 2015) studies. So far, digital traces on many tools and platforms have been studied, including Twitter (Haustein, Peters, Sugimoto, Thelwall, \& Larivière, 2014c), Mendeley (Thelwall \& Wilson, 2015), ResearchBlogging (Shema, Bar-Ilan \& Thelwall, 2014 ), F1000 (Mohammadi \&

\footnotetext{
* Corresponding author.

E-mail address: shenmeng@email.unc.edu (S. Xu).
} 


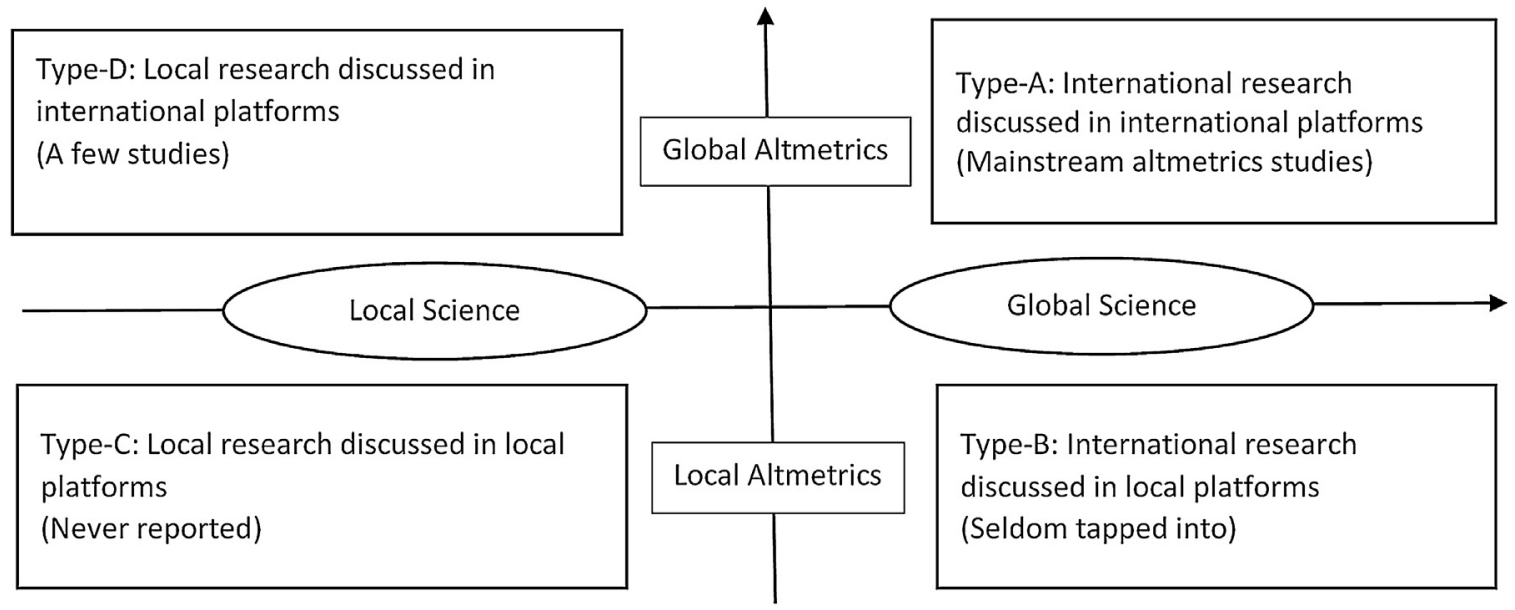

Fig. 1. Four types of altmetrics studies.

Thelwall, 2013), ResearchGate (Thelwall \& Kousha, 2015) and YouTube (Kousha, Thelwall \& Abdoli, 2012), among others. While scientific article is still the most commonly studied type of scientific product, many other types are explored, for instance, blogs (Shema, Bar-Ilan \& Thelwall, 2012), software (ImpactStory, 2016), slides (Kraker, Lex, Gorraiz, Gumpenberger, \& Peters, 2015), datasets (Peters et al., 2015), and videos (Kousha et al., 2012). Altmetrics has been applied in scientific evaluation. Evaluated objects can be a journal (Loach \& Evans 2015), an institution (Peters et al., 2014; Rehemtula, Rosa, Leitao, \& Avilés, 2014), a discipline (Holmberg \& Thelwall 2014) or a scientist (Kolahi, 2015). Some institutions (ScienceOpen, 2016) have enabled users to rank literature retrieval results by the Altmetric Attention Score.

Altmetrics has drawn attention from worldwide academia. As shown in Fig. 1, four types of altmetrics research are defined based on the geographical variation of altmetrics sources and scientific outputs. Type-B and type-D altmetrics studies investigate the communication between local science and global science, while type-C altmetrics studies mainly focus on domestic scientific communication. Alperin (2013) argues that by disseminating research in online social media, the altmetrics movement would reduce the bias caused by leading bibliographic databases where research from developing countries is underrepresented. However, altmetrics research hitherto has been focused on type-A studies where data are collected from international platforms, of research from international mainstream publishers, prevailingly in developed countries. A few researchers have considered local altmetrics and conducted Type-D studies. Alperin (2015) investigated the coverage of altmetrics data in the prominent Latin American journal portal SciELO and found the coverage level of most social media sources was zero or negligible. Poplasen (Poplašen \& Zrnić 2014) tried to use altmetrics for measuring science in Croatia. Tammaro (2014) tested altmetrics as an evaluation method for Italian scholars in the humanities. Torres et al. (Torres, Cabezas, \& Jimenez, 2013) conducted a case study on a sample of Spanish communication studies. However, these studies all used data from international social media platforms, neglecting local platforms, which play an important role in domestic scientific communication.

Type-B and type-C studies are seldom seen, because the infrastructure for analyzing local altmetrics, for example data aggregating services, is not well established. This paper argues that local altmetrics is an integral part of the holistic altmetrics landscape. Discussions on local altmetrics sources can function as channels for introducing international scientific research. This is true especially in countries where English is not the first language or where global platforms are blocked. Including local altmetrics of different context would reveal a more comprehensive view of a research product's true impact over the world.

China, while thriving as a strong scientific power, has restricted access to many international social media platforms such as Twitter, Facebook, and YouTube. Sina Weibo is currently the most widely used microblog service. As a type-B study, the aim of this study is twofold: (1) To investigate the characteristics of an important local altmetrics source, namely Weibo, in discussing global science. It is of particular interest to see how global scientific research is discussed on Weibo by Chinese users, considering that China is a large economic entity which also has a large academic community. (2) To study the difference between Weibo and Twitter as a comparison of local altmetrics and global altmetrics. Although both of these two platforms are popular microblog services, they have completely different users from diverse cultural backgrounds. It is also of significant meaning to compare altmetrics based on these two platforms to reveal the nature of altmetrics of microblog services as a whole.

\section{Research questions}

The research questions are: (1) How frequently do Sina Weibo users discuss global science? (2) How immediate is Weibo altmetrics? In other words, how soon do weibos appear after the publication of the articles? (3) What are the distributions 
of Weibo altmetrics on the article, source and discipline levels? How are the patterns compared with Twitter altmetrics? (4) How and why do Weibo users discuss global scientific articles? To answer this question, we mainly focus on the motivation, elements mentioned, and sentiment of the top weibos.

This study contributes to altmetrics studies in four ways: (1) We study a novel altmetrics, Weibo, and call for attention on local altmetrics. It will advance the development of domestic altmetrics and take altmetrics studies one step further to provide a more complete view of research impact. (2) We measure the immediacy of microblog altmetrics in a systematic way. The result provides evidence of whether and how much microblog altmetrics is faster than citations. (3) We conduct a comprehensive comparison between Weibo and Twitter. As the two most popular microblog services, the similarities and differences between them in global science discussion are of particular interest to scientific communication. (4) Moreover, in this study, we use the largest altmetrics dataset of Weibo and Twitter so far. Our results will provide reference to other related empirical studies.

\section{Methodology}

\subsection{Used terms}

For convenience of discussion, frequently used terms in the paper are explained as follows.

Altmetrics: According to the context, this could refer to the research field or the altmetrics indicators.

Weibo: The proper noun Weibo refers to the microblog platform Sina Weibo (Weibo.com). The common noun weibo refers to a microblog posted on Sina Weibo, i.e., a Weibo post. When used as a verb, weibo refers to the behavior of posting a microblog on Weibo.

Weibo altmetrics: This denotes altmetrics based on Sina Weibo. When a research product is mentioned in a Weibo via a link, a DOI or other trackable ways, we say that this research product receives a Weibo mention, or has Weibo altmetrics.

Scientific weibo: We refer to a weibo as a scientific weibo when it mentions a scientific product and usually contains a link to it.

\subsection{A brief view of Weibo}

Weibo, originally the Chinese Pinyin for microblog and referring to general microblog services, was branded by Sina Corporation in 2010. Now Weibo normally refers to Sina Weibo. As microcosm of Chinese society, Weibo has attracted a wide range of users, including celebrities and public figures, organizations such as media outlets, businesses, government agencies and charities, as well as the general public. According to the latest report, in November 2015, Weibo had 261 million monthly active users, compared with 310 million monthly active Twitter users. Weibo enables users to express and share their ideas, opinions and stories in the form of text and attached multimedia, including images, music, and short videos. There used to be a 140 character limit on Weibo posts, however, the limit was removed in February 2016.

A dominating $97.2 \%$ of Weibo users are from China, however, only $21.9 \%$ of Twitter users are from the U.S. In this sense, Sina Weibo is a local microblogging service in China. Like Twitter, Weibo is widely used for scientific communication. It helps evaluate the quantity and quality of information flux between traditional scientific expert communities and the broader public (Zhao, Chen, Ge, Yu, \& Shao, 2014). One report shows that Weibo users who are engaged in science include professional researchers, teachers in colleges and universities, science writers, journalists, and editors of science, etc. (Liu, 2012).

\subsection{Dataset}

Altmetric.com began to track Weibo in March 2014 upon customers' request, and the tracking ended in July 2015 (Altmetric LLP, 2016), because the cost became unaffordable when Sina Weibo stopped distributing data to external companies and ever since started up using Socialgist as their sole non-Chinese data broker. ${ }^{1}$ Altmetric.com provided the full dataset from October 2011 to November 2015 for this research, making it possible to conduct large scale comparison and analysis. The dataset contained over 4.4 million records in JSON format. A relational database was established for retrieval's convenience, and python scripts were used to extract and analyze the data.

To compare Weibo altmetrics with other altmetrics in the same time window, we focused on records captured by Altmetric.com from March 2014 to July 2015. The total number of records was 1.99 million. For specific research purposes, for example the immediacy analysis of Twitter, records of the entire period (i.e. October 2011 to November 2015) were used. In the dataset, each record represents a research product of which altmetrics activities are captured. The altmetrics include those based on Weibo, Twitter, Facebook, Blog, Wikipedia, News, Google Plus, Policy, Reddit, F1000, Peer Reviews, Video, and Q\&A. As will be demonstrated in Section 3.1, these altmetrics have various levels of coverage on articles. A brief description of these indicators can be found in Table 1.

\footnotetext{
1 Personal communication with Euan Adie.
} 


\begin{tabular}{ll}
\hline Indicator & Description \\
\hline Twitter & Number of mentions an article receives on Twitter \\
Weibo & Number of mentions an article receives on Weibo \\
Facebook & Number of mentions an article receives on Facebook walls \\
Blog & Number of mentions an article receives on over 9000 academic and non-academic blogs feeds \\
& tracked via RSS by Altmetric.com \\
Wikipedia & Number of mentions an article receives on Wikipedia.org \\
News & Number of mentions an article receives on over 2000 mainstream media \\
Google Plus & Number of mentions an article receives on Google Plus \\
Policy & Mentions of publications in policy documents. According to Liu, Konkiel, \& Williams (2015), \\
& policy documents are from diverse groups such as the International Monetary Fund, World \\
Reddit & Health Organization, and Intergovernmental Panel on Climate Change. \\
F1000 & Number of mentions an article receives on Reddit \\
Peer Reviews & Article factor calculated from the recommendations for a publication on F1000 \\
Video & Evaluations of individual outputs from contributor to open post-publication peer review \\
Q\&A & forums Pubpeer and Publons \\
& Number of mentions an article receives in video descriptions and comments on YouTube \\
& Mention of an article in questions and answers on Stack Overflow (stackoverflow.com) \\
\hline
\end{tabular}

\subsection{Coverage calculation}

Coverage statistics reflects the activeness of altmetrics indicators. For example, if $90 \%$ of a set of articles have been tweeted but only $10 \%$ of them are saved in Mendeley, then the coverage of Twitter altmetrics is higher than Mendeley altmetrics for this set of articles. The coverage of altmetrics have been discussed in a number of studies. For instance, Araújo, Murakami, Lara, and Fausto (2015) examined Twitter and Facebook mentions of articles published in a Brazilian LIS journal; Haustein, Bowman, Macaluso, Sugimoto, and Larivière (2014) explored Twitter activities and Altmetric coverage of articles on Arxiv; Zahedi, Costas, and Wouters (2014) studied the presence and distribution of altmetrics in the set of publications, across fields, and document types; Haustein et al. (2014b) investigated the adoption of altmetrics sources by sampled bibliomatricians. While these results provide some idea about the activeness of altmetrics, the coverages they have explored are under different contexts, making it difficult to compare them. Our study, therefore, calculates the relative coverage of Weibo altmetrics, along with several other popular altmetrics. We use the total number of research records (1.99 million) tracked by Altmetric.com in the Weibo data collection period (from March 2014 to July 2015) as the denominator, and the number of records for each altmetrics source as the numerator, to calculate the percentage. In this way, despite the total coverage of Altmetric.com, we can have a better understanding of the relative coverage of Weibo altmetrics, compared to others.

\subsection{Immediacy calculation}

The time difference between the publication date and the first Weibo mention date is calculated to measure the immediacy of Weibo altmetrics. The same measuring technique is used on Twitter and the general altmetrics. As discussed by Haustein, Bowman, and Costas (2015), the first public online appearance of VoR (Version of Record) should be used as the basic time unit to determine the official publication date of a paper, and among many available data sources, the publication date collected by Altmetric.com, which is a mixture of journal issue date and online date, is one of the best proxies for online publication. As a result, Altmetric publication date was used as the publication date in our immediacy calculation. Let $T_{p w}$ denote the time $T_{w}$ be the first Weibo mention date, $T_{p}$ be the Altmetric publication date, then

$$
T_{p w}=T_{w}-T_{p} \text { (days) }
$$

If $T_{p w}<180$ days, the Weibo mention is defined to be immediate, compared with citations that would take years to accumulate (Brody \& Harnad, 2006). The immediacy distribution is presented in time intervals of 1 day, 7 days (one week), 30 days (one month), 180 days (half year), 360 days (one year) and over 360 days. When the first Weibo mention is prior to the Altmetric publication date, $T_{p w}$ is negative. For comparison, the immediacy of Twitter and the general altmetrics, measured respectively by $T_{p t} T_{p a}$ defined the same way as $T_{p w}$, were also calculated. $T_{a}$ is the first date when an article is captured by any altmetrics source tracked by Altmetric.com.

\subsection{Distribution calculation and classification schema for disciplines}

To study the distribution of Weibo altmetrics, we count the number of Weibo posts for each article, each source and each discipline. As observed, Altmetric.com has maintained the discipline category based on the Scopus schema $\left(S_{S}\right)$ and two other classification systems, i.e. publisher discipline $\left(S_{p}\right)$ and Medline discipline $\left(S_{m}\right) .48 .2 \%$ of the weiboed articles have all three classification schemes, while $78.7 \%$ of articles have at least one of these three classification code. Specifically, $S_{S}$ covers the most articles (72.8\%), $S_{p}$ covers the second most articles (68.7\%), and $S_{m}$ covers the least articles (59.5\%). $21.3 \%$ of articles are not classified. These articles are mainly either from open access or preprint platforms such as arXiv, SSRN, IEEE and so 
Table 2

Motivation coding scheme.

\begin{tabular}{|c|c|c|}
\hline First level code & Second level code & Definition \\
\hline \multirow[t]{7}{*}{1 Dissemination } & 1.1 Dissemination -Interesting & To highlight the interesting part(s) of the article to attract attention \\
\hline & 1.2 Dissemination -Surprising & $\begin{array}{l}\text { To highlight the surprising fact(s) beyond normal expectation to attract } \\
\text { attention }\end{array}$ \\
\hline & 1.3 Dissemination -Academically useful & To highlight the academic usefulness of the article to attract attention \\
\hline & 1.4 Dissemination -Practically useful & To highlight the practical usefulness of the article to attract attention \\
\hline & 1.5 Dissemination - Link only & To provide the link \\
\hline & 1.6 Dissemination -Perfunctory introduction & To provide the link with very brief, perfunctory introduction \\
\hline & 1.7 Dissemination -Request for access & To ask for help with the access of the article \\
\hline \multirow[t]{7}{*}{2 Discussion } & 2.1 Discussion - Interesting & $\begin{array}{l}\text { To elaborate the interesting part(s) of the article to arouse interactive } \\
\text { communication }\end{array}$ \\
\hline & 2.2 Discussion -Surprising & $\begin{array}{l}\text { To elaborate the surprising fact(s) of the article beyond normal expectation to } \\
\text { arouse interactive communication }\end{array}$ \\
\hline & 2.3 Discussion -Academically useful & $\begin{array}{l}\text { To elaborate the academic value of the article to arouse interactive } \\
\text { communication }\end{array}$ \\
\hline & 2.4 Discussion -Practically useful & $\begin{array}{l}\text { To elaborate the practical value of the article to arouse interactive } \\
\text { communication }\end{array}$ \\
\hline & 2.5 Discussion -Supporting a claim & To reference the article to support a claim \\
\hline & 2.6 Discussion -Criticizing & To criticize the article \\
\hline & 2.7 Discussion - Responding & To respond to relevant reports of the article \\
\hline \multirow[t]{2}{*}{3 Marketing } & 3.1 Marketing -Self-promotion & To promote one's own work \\
\hline & 3.2 Marketing -Other promotion & To promote others' work \\
\hline \multirow[t]{2}{*}{4 Triggering } & 4.1 Triggering -Reuse & To save for future reuse \\
\hline & 4.2 Triggering-Association & To relate the article to relevant ideas \\
\hline
\end{tabular}

on, or from other small scale publishers, who maintain their own classification system that are, however, too sparse to be recorded and analyzed.

Considering that $S_{S}$ has the highest coverage, here in this study we use it in the discipline level analysis. It must be noticed that $S_{S}$ is slightly different from the current Scopus classification schema. The current Scopus classification has divided "Physics and Astronomy" into two smaller categories, i.e., "Astronomy, Astrophysics, Space Science" and "Physics". $S_{S}$ has 30 disciplines of which the abbreviation and full title can be referenced in Appendix A. Publisher $S_{p}$ mainly consists of the classification systems adopted by the ERA (Excellence in Research for Australia), the NPG (Nature Publishing Group), and Springer. Specifically, ERA has 155 disciplines in its classification system, Springer has 250, and NPG has 882, according to the Altmetric.com data. Amidst weiboed articles covered $S_{p}$, ERA articles take up $76.6 \%$, NPG articles take up $17.5 \%$ and Springer articles take up 6.9\%. The NPG's disciplines are found to be more like keywords rather than disciplines. Due to the heterogeneous nature of $S_{p}$; publisher discipline was used for reference. Medline discipline $S_{m}$ provides detailed disciplines of general medical science and has 96 disciplines. It could provide insight in the further analysis of disciplinary distribution of the medical field. A good combination of these three classification systems can clearly reveal the disciplinary distribution of the weiboed articles.

\subsection{Content analysis}

In order to understand why users weibo the highly weiboed scholarly articles, content analysis was conducted on weibos of the top $1 \%$ ( 109 of 10,754 ) of weiboed articles, which accounts for $29 \%(10,775$ of 37,200$)$ of the total weibos. Given that reposts do not disclose enough information for the analysis of motivation, in this study we focus on original weibos, which occupy $41 \%$ of the total. For each of the 109 highly weiboed articles, 5 original weibos were randomly selected. If for an article the number of original weibos is less than 5, all original weibos of this article were collected. As a result, 321 original weibos were sampled and analyzed.

In the first step, we referenced the coding scheme of Na's work (Na, 2015) when analyzing motivation for tweeting scholarly articles to form a basic idea of the motivation categories. 50 weibos were then coded by three coders. After discussion, the initial coding scheme was formed. In the second step, another 50 weibos were coded using the initial scheme. The agreement rate was $72 \%$. Three coders discussed again, with a particular focus on the coding disagreement and potential new categories. The initial scheme was then revised to the final version of coding scheme. In the third step, two coders coded all 321 weibos. The agreement rate was $87 \%$. The disagreed weibos were coded again by the third coder.

Mainly three aspects of weibo content were analyzed. The coding schema are presented in Tables $2-4$. All weibos were manually coded.

(1) Motivation. Four major motivation categories are identified, which are dissemination, discussion, marketing and triggering. Dissemination has the intent of informing more people about an article, usually by providing the link of it without further explanation, or briefly mentioning highlights of the article in order to attract attention; Discussion has the intent of making certain points in an article clearer, usually by describing, reasoning, applying, summarizing, commenting or 
Table 3

Element coding scheme.

\begin{tabular}{ll}
\hline Code & Definition \\
\hline 1 Title & Title of the article \\
2 Abstract & Abstract of the article \\
3 Methodology & Methodology of the article \\
4 Conclusion & Conclusion of the article \\
5 Concept & Terms or other concepts in the article \\
6 Fragment & A piece of content in the article (a number, a figure or a sentence) \\
7 Topic & Main topic of the article \\
8 Summarize & Brief summarization of the article \\
9 Overall & General feeling of the article \\
10 Indirect mention & Mentions indirect sources (e.g., a report talking about the article) \\
11 Pure link & Mentions no element of the article \\
\hline
\end{tabular}

Table 4

Sentiment coding scheme.

\begin{tabular}{ll}
\hline Code & Description \\
\hline 1 Neutral & When weibo shows no clear positive or negative attitude towards the article \\
2 Positive & When weibo shows a clear positive attitude towards the article \\
3 Negative & When weibo shows a clear negative attitude towards the article \\
\hline
\end{tabular}

Table 5

Relative coverage of Weibo compared with other altmetrics sources.

\begin{tabular}{|c|c|c|c|c|c|c|c|c|}
\hline Altmetrics & No. & Percentage & Altmetrics & No. & Percentage & Altmetrics & No. & Percentage \\
\hline Twitter & 1308015 & $65.7 \%$ & Google Plus & 48965 & $2.5 \%$ & Peer Reviews & 9188 & $0.5 \%$ \\
\hline Facebook & 347256 & $17.5 \%$ & Policy & 17638 & $0.9 \%$ & Video & 8293 & $0.4 \%$ \\
\hline Blogs & 123656 & $6.2 \%$ & Reddit & 16300 & $0.8 \%$ & $\mathrm{Q} \& \mathrm{~A}$ & 2983 & $0.2 \%$ \\
\hline Wikipedia & 122309 & $6.2 \%$ & F1000 & 11354 & $0.6 \%$ & & & \\
\hline News & 119759 & $6.0 \%$ & Weibo & 10754 & $0.5 \%$ & & & \\
\hline
\end{tabular}

criticizing specific aspects of the article; Marketing is when an article is praised and recommended explicitly; Triggering is when an article reminds the Weibo user of something relevant. (See Table 2.)

(2) Element. Element mentioned in the Weibo is coded to demonstrate which part of articles attracts the most Weibo attention. (See Table 3.)

(3) Sentiment. Sentiment of the weibos indicates whether the user has a positive, neutral, or negative attitude towards the article mentioned. (See Table 4.)

\subsection{Limitation}

This study considers only articles among all available types of research products, and heavily relies on the accuracy of data collection by Altmetric.com. However, Altmetric LLP is currently the only Weibo tracker, and has the most professional article tracking experience (Zahedi, Fenner \& Costas, 2014). Therefore, the dataset is considered complete and valid enough to explore the research questions.

When calculating the immediacy of Weibo altmetrics, Altmetric publication date is used as the proxy of the first public online appearance of VoR, because there is hardly a systematic way of collecting the ideal publication date. This bottleneck awaits better solution by making various dates reported by publishers more transparent and standardized (Haustein et al., 2015).

\section{Results}

\subsection{Coverage of Weibo altmetrics}

According to Table 5 , the coverage of Weibo is $0.5 \%$, which is significantly dwarfed by Twitter, which has a coverage of $65.7 \%$. Facebook (17.5\%), blogs (6.2\%), Wikipedia (6.2\%) and News (6.0\%) are also popular altmetrics sources. The only three sources having lower coverage than Weibo are Peer Reviews, Video and Q\&A. Policy, Reddit and F1000 are of similar coverage level $(<1 \%)$ as Weibo. The result shows that although Altmetric.com so far only tracks articles from the international academia, Weibo users are communicating global science in the Chinese society. 

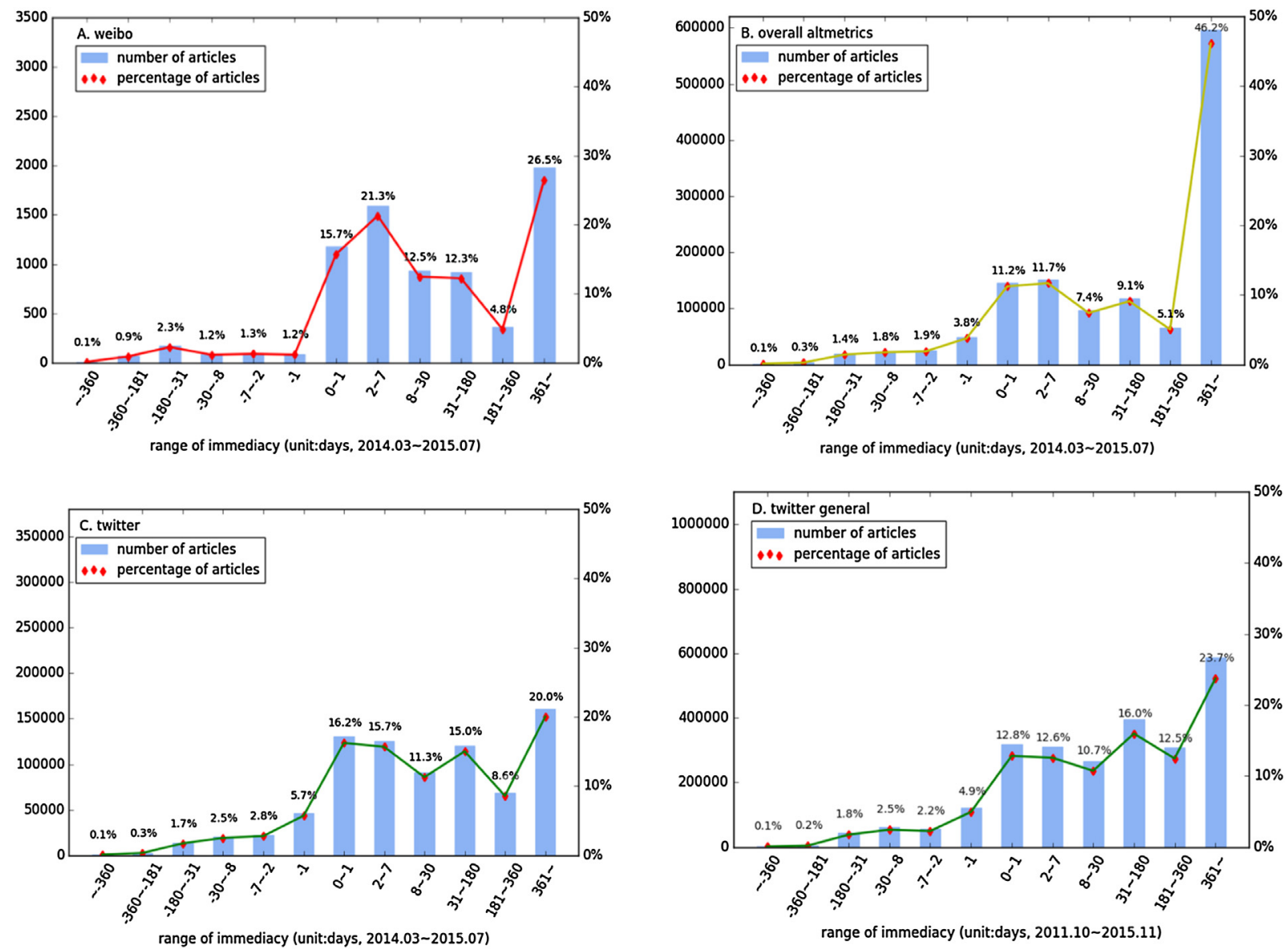

Fig. 2. Immediacy distribution of Weibo altmetrics.

\subsection{Immediacy of Weibo altmetrics}

Altmetrics was proposed to be more immediate than citations, which usually take months or years to accumulate (Priem, Taraborelli, Groth, \& Neylon, 2010). To confirm this, we defined and calculated the immediacy of Weibo posts, as well as Twitter and the general altmetrics. Results are shown in Fig. 2. Regarding Weibo, Fig. 2-A shows that Weibo altmetrics is more immediate compared with citations, in that $69 \%$ of articles with Weibo attention are captured within 180 days. It's highlighted that $44 \%$ of articles in the dataset get their first Weibo post in no more than 7 days, and particularly, $7 \%$ of them have Weibo posts prior to their formal publication. Still, $27 \%$ of the articles receive fairly lagged ( $T_{p w}>360$ days) Weibo attention, implying that Weibo users also discuss old articles. The general altmetrics respond more slowly than Weibo. As Fig. 2-B illustrates, $46 \%$ of general altmetrics happen more than 360 days after publishing. This implies that altmetrics sources have different levels of immediacy, and some of them may not be as immediate as expected.

Next, we compared the immediacy of Weibo with Twitter. In Fig. 2-C, it can be seen that Twitter shares a similar immediacy distribution with Weibo, reflected in that $64 \%$ of tweeted articles are firstly tweeted within 180 days, $37 \%$ of them receive their first tweet within 7 days, and particularly, $12 \%$ are tweeted before formal publishing. Fig. 2-D is the immediacy distribution of Twitter in a broader time span, showing a slight drop in time interval of [0,1] and (1,7], and a bit of rise in [180,360] and (360,). Nevertheless, the shape of the curve is basically the same with Fig. 2-C, demonstrating that the distribution is stable and reflects the nature of Twitter altmetrics.

\subsection{Distribution of Weibo altmetrics on the article level}

Citation distribution is well known for its skewness (Seglen, 1992). Therefore, we investigated whether Weibo altmetrics follows a similar distribution. Fig. 3 is the cumulative post counts distribution of Weibo in comparison with Twitter. As is seen, the distribution is heavily skewed. $10 \%$ of the articles are mentioned by $61 \%$ of the weibos, and $20 \%$ of the articles are discussed in $72 \%$ of the weibos. The skewness is even more significant in Twitter altmetrics' distribution, with $10 \%$ of the articles mentioned in $66 \%$ of the tweets and $20 \%$ articles discussed in $76 \%$ of the total tweets. Fig. 4 compares the distribution 


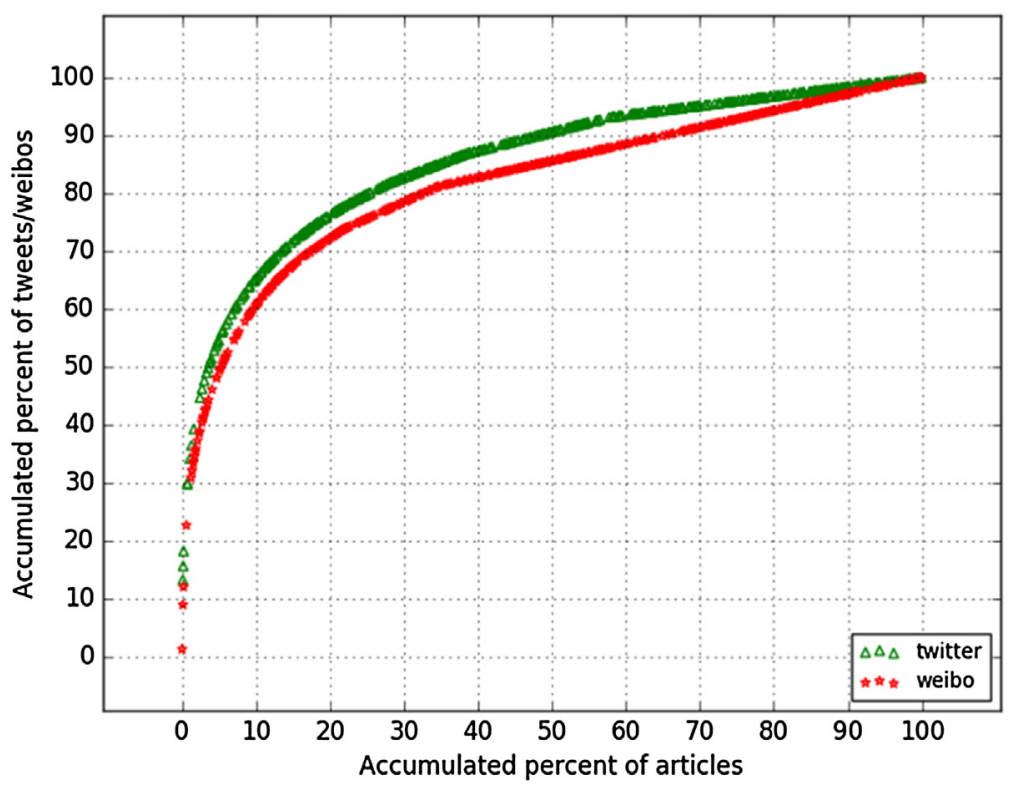

Fig. 3. Distribution of Weibo vs. Twitter.

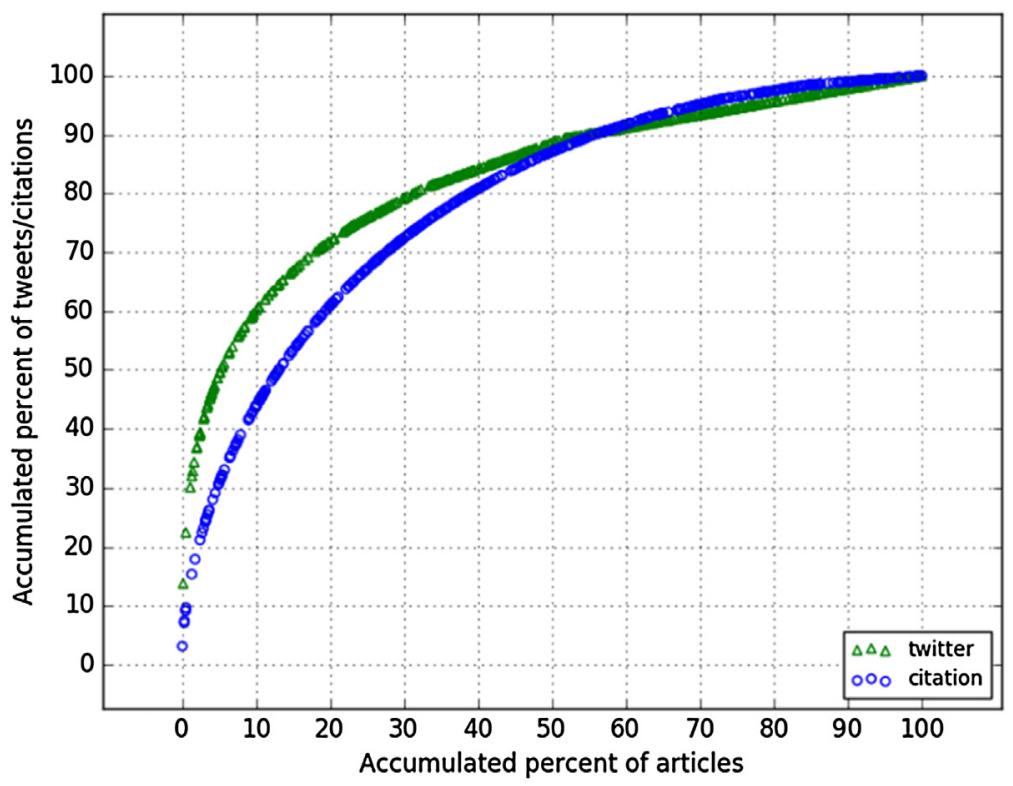

Fig. 4. Distribution of Twitter vs. citation.

of Twitter altmetrics and citation, using Scopes citation data and Altmetric.com data over three periods (i.e. January 2012, January 2013 and January 2014). A detailed description of the dataset can reference (Yo, 2016a). As shown in Fig. 4, 10\% of journal articles accumulate only around $42 \%$ of total citations, and $20 \%$ of journal articles account for about $61 \%$ of overall citations. Hence, the overall skewness of microblog altmetrics is higher than citations.

Fig. 5 illustrates Weibo altmetrics distribution from another perspective. Weibo has $65.5 \%$ of articles with only 1 post, $12.9 \%$ of articles with 2 posts, and $5.5 \%$ of articles with 3 posts. In other words, $83.9 \%$ of the articles obtain no more than 3 posts if they receive any Weibo attention. Compared with Weibo, Twitter has less articles that get tweeted only once (44.4\%), but more articles that get tweeted twice (19.2\%). In total, $73.6 \%$ of the articles are tweeted no more than 3 times. The distribution demonstrates a low level of both Weibo and Twitter altmetrics for most articles. Moreover, not a single article gets more than 500 Weibo posts; Whereas, a small portion of articles receive over 500 tweets, and in rare cases, the number of tweets could reach 17,679. On average, a weiboed article receives 3.5 weibos, and a tweeted article receives 6.2 tweets. 


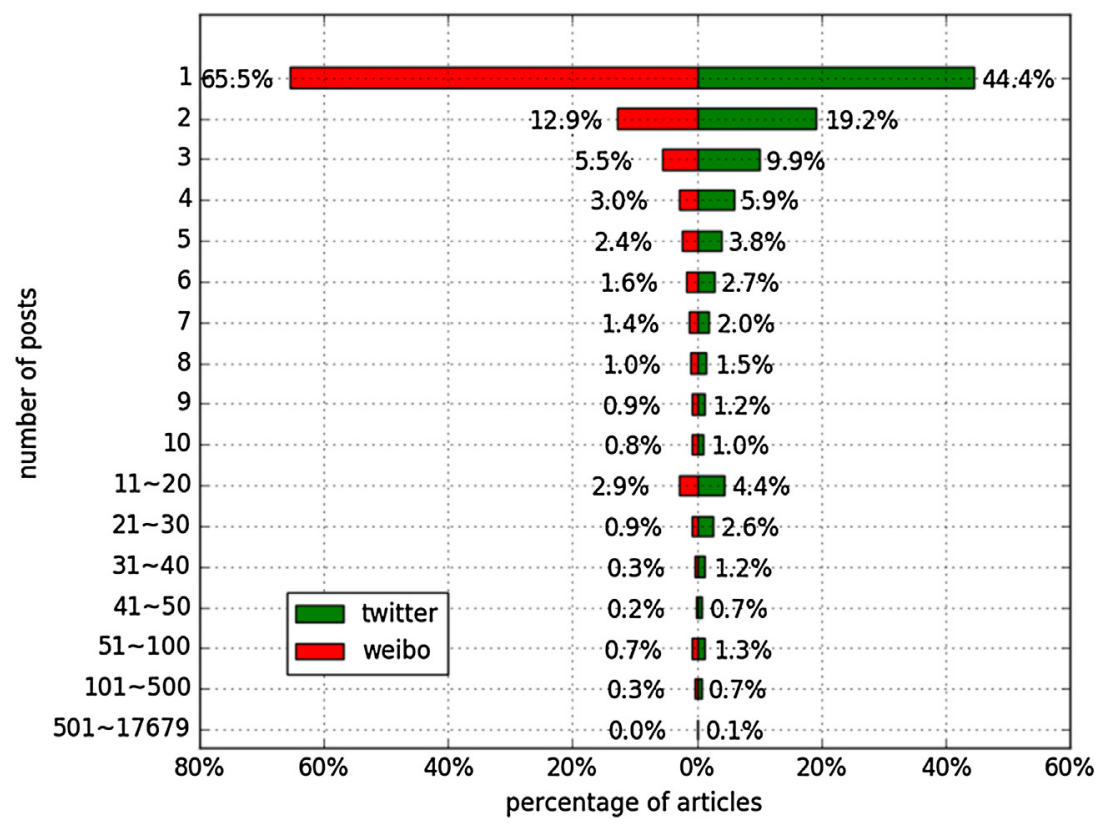

Fig. 5. Frequency distribution of Weibo vs. Twitter.

Table 6

Motivation coding result.

\begin{tabular}{|c|c|c|}
\hline Code & No. & Percentage \\
\hline 1.1 Dissemination -Interesting & 18 & $5.6 \%$ \\
\hline 1.2 Dissemination -Surprising & 10 & $3.1 \%$ \\
\hline 1.3 Dissemination -Academically useful & 11 & $3.4 \%$ \\
\hline 1.4 Dissemination -Practically useful & 7 & $2.2 \%$ \\
\hline 1.5 Dissemination - Link only & 17 & $5.3 \%$ \\
\hline 1.6 Dissemination -Perfunctory introduction & 67 & $20.9 \%$ \\
\hline 1.7 Dissemination - Request for access & 1 & $0.3 \%$ \\
\hline 1 Overall dissemination & 131 & $40.8 \%$ \\
\hline 2.1 Discussion -Interesting & 24 & $7.5 \%$ \\
\hline 2.2 Discussion -Surprising & 33 & $10.3 \%$ \\
\hline 2.3 Discussion -Academically useful & 37 & $11.5 \%$ \\
\hline 2.4 Discussion -Practically useful & 33 & $10.3 \%$ \\
\hline 2.5 Discussion -Supporting a claim & 23 & $7.2 \%$ \\
\hline 2.6 Discussion -Criticizing & 3 & $0.9 \%$ \\
\hline 2.7 Discussion - Responding & 12 & $3.7 \%$ \\
\hline 2 Overall discussion & 165 & $51.4 \%$ \\
\hline 3.1 Marketing -Self-promotion & 3 & $0.9 \%$ \\
\hline 3.2 Marketing -Other promotion & 17 & $5.3 \%$ \\
\hline 3 Overall marketing & 20 & $6.2 \%$ \\
\hline 4.1 Triggering -Reuse & 1 & $0.3 \%$ \\
\hline 4.2 Triggering -Association & 4 & $1.3 \%$ \\
\hline 4 Overall triggering & 5 & $1.6 \%$ \\
\hline
\end{tabular}

\subsection{Content analysis of Weibo altmetrics}

Results of the content analysis of the top $1 \%$ of weibos are shown in Tables 6, 7 and 9 . In addition, statistical result of how motivation and element co-occur is shown in Table 8.

Table 6 shows that the most common motivation for weiboing an article is to disseminate this article by providing both a link and some perfunctory introduction (code 1.6, 20.9\%). It is followed by to discuss by elaborating the academic usefulness of the article (code $2.3,11.5 \%$ ), to discuss by elaborating the practical usefulness of the article (code $2.4,10.3 \%$ ) and to discuss by elaborating the surprising part(s) (code 2.2,10.3\%) or interesting part(s) (code 2.1, 7.5\%) of the article. Meanwhile, a small percentage of marketing (6.2\%) and triggering (1.6\%) are observed. In general, discussion is the major motivation category (51.4\%).

Table 7 shows that a large percentage of weibos (code 11,33\%) mention no element of the articles. The most commonly mentioned element of articles in Weibo is the conclusion (code 4, 23.1\%). It's followed by the main topic of the article (code $7,14.3 \%$ ) and fragment of content (code 6, 8.7\%). 
Table 7

Element coding result.

\begin{tabular}{lll}
\hline Code & NO. & Percentage \\
\hline 1 Title & 19 & $5.9 \%$ \\
2 Abstract & 1 & $0.3 \%$ \\
3 Methodology & 18 & $5.6 \%$ \\
4 Conclusion & 73 & $23.1 \%$ \\
5 Concept & 1 & $0.3 \%$ \\
6 Fragment & 28 & $8.7 \%$ \\
7 Topic & 46 & $14.3 \%$ \\
8 Summarize & 17 & $5.3 \%$ \\
9 Overall & 9 & $2.8 \%$ \\
10 Indirect mention & 2 & $0.6 \%$ \\
11 Pure link & 106 & $33.0 \%$ \\
\hline
\end{tabular}

Table 8

Distribution of motivation-element pair (NO.>3).

\begin{tabular}{|c|c|c|c|c|c|c|c|c|c|c|c|}
\hline M. & E. & NO. & Percentage & M. & E. & NO. & Percentage & M. & E. & NO. & Percentage \\
\hline 1.6 & 11 & 45 & $14.0 \%$ & 2.3 & 8 & 9 & $2.8 \%$ & 2.5 & 4 & 5 & $1.6 \%$ \\
\hline 2.4 & 4 & 25 & $7.8 \%$ & 1.1 & 11 & 7 & $2.2 \%$ & 2.3 & 3 & 4 & $1.3 \%$ \\
\hline 2.1 & 4 & 16 & $5.0 \%$ & 2.3 & 7 & 7 & $2.2 \%$ & 2.3 & 11 & 4 & $1.3 \%$ \\
\hline 2.2 & 6 & 15 & $4.7 \%$ & 1.1 & 7 & 6 & $1.9 \%$ & 2.1 & 7 & 4 & $1.3 \%$ \\
\hline 1.5 & 11 & 15 & $4.7 \%$ & 2.5 & 11 & 6 & $1.9 \%$ & 2.4 & 9 & 4 & $1.3 \%$ \\
\hline 1.6 & 1 & 14 & $4.4 \%$ & 2.2 & 7 & 5 & $1.6 \%$ & 1.2 & 7 & 4 & $1.3 \%$ \\
\hline 3.2 & 11 & 13 & $4.1 \%$ & 2.5 & 3 & 5 & $1.6 \%$ & 1.4 & 11 & 4 & $1.3 \%$ \\
\hline 2.2 & 4 & 11 & $3.4 \%$ & 1.6 & 7 & 5 & $1.6 \%$ & 1.3 & 3 & 4 & $1.3 \%$ \\
\hline 2.3 & 4 & 10 & $3.1 \%$ & 1.2 & 6 & 5 & $1.6 \%$ & & & & \\
\hline
\end{tabular}

*M. is motivation; E. is element; NO. is the number of combination of M. and E.; Percentage. is the percentage of the combination in all possible combinations. For the code number, please refer to Tables 6 and 7.

Table 9

Sentiment coding result.

\begin{tabular}{lll}
\hline Sentiment & NO. & Percentage \\
\hline 1 Neutral & 272 & $84.7 \%$ \\
2 Positive & 43 & $13.4 \%$ \\
3 Negative & 6 & $1.9 \%$ \\
\hline
\end{tabular}

Table 8 shows the pattern of how the motivation categories co-occur with the element categories. It is common to see in weibos that an article link with perfunctory introduction co-occur with no mention of any element of the article (14\%). Plenty of weibos discuss the conclusions of the article because they are practically useful (7.8\%) or interesting (5\%). Users also mention fragment of content in the article because they find it beyond normal expectation (4.7\%).

Table 9 shows that most of weibos are neutral (84.7\%), certain percentage are positive (13.4\%), and very few are negative (1.9\%).

\subsection{Distribution of Weibo altmetrics on the source level}

In citation analysis, Garfield's law of concentration (Garfield, 1998) indicates that a few core journals receive most of the citations. In our study, we include the source distribution of Weibo metrics in Fig. 6. Fig. 6-A highlights the coverage of the top weiboed sources. The most frequently weiboed source alone has $8.2 \%$ of all the Weibo posts, and the 10 top weiboed sources has $30.6 \%$ of the total Weibo posts. Twitter shows a similar phenomenon, with the most frequently tweeted source accounting for $10.3 \%$ of the total tweets. Nevertheless, the increasing speed of cumulative percentage afterwards is lower than Weibo, due to the large number of tweeted sources. Fig. 6-B shows the distribution of mentioned articles over percentage of sources, rather than the absolute number. Curves of Weibo and Twitter meet at point (5.5\%, 62.3\%). Before this point, the Weibo curve is above Twitter curve, which means that the same percentage of mentioned sources have a higher percentage of posts than Twitter. In other words, Weibo altmetrics has a more concentrated core source area. The overall concentration degree of Weibo altmetrics is higher than citations, as less than $10 \%$ of the sources ( 200 sources) cover $68 \%$ of the total weiboed articles, whereas Garfield's study (Garfield, 1972) showed $10 \%$ sources received $51 \%$ of all the citations. Fig. 6-C examines the distribution using Bradford's method (Vickery, 1948), where journals are ranked in descending order by number of posts. $\mathrm{N}$ is the cumulative number of journals, and $\mathrm{R}(\mathrm{n})$ is the cumulative number of posts. According to Bradford's law, the distribution curve consists of three parts: an exponential increase representing the core journals, followed by a linear increase representing relevant journals, and a logarithmic increase representing irrelevant journals. As shown in Fig. 6-C, the Weibo curve is linear, while the Twitter curve shows clearly three separate parts. In other words, Twitter altmetrics follows the traditional Bradford's law, and the tweeted sources can be classified into three sections. Meanwhile, 2412 sources were 

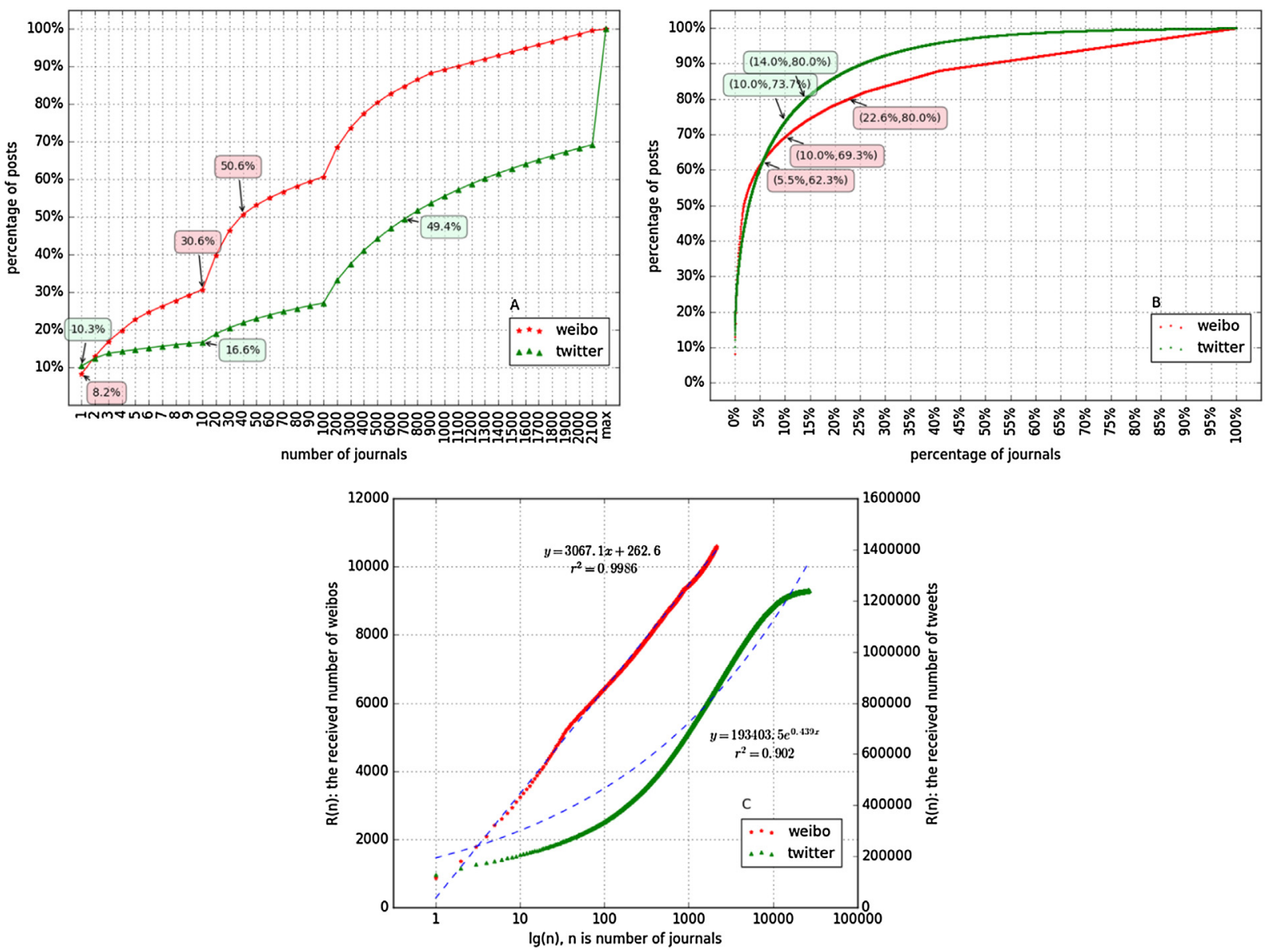

Fig. 6. Source distribution of Weibo vs. Twitter.

Table 10

Top weiboed sources vs. top tweeted sources (top 15).

\begin{tabular}{|c|c|c|c|c|c|}
\hline No. & weiboed source & $N_{w}$ & No. & Tweeted source & $N_{t}$ \\
\hline 1 & Nature & 866 & 1 & arXiv & 127413 \\
\hline 2 & arXiv & 495 & 2 & PLOS ONE & 25678 \\
\hline 3 & Science & 421 & 3 & SSRN & 16308 \\
\hline 4 & Cell & 319 & 4 & Nature & 6169 \\
\hline 5 & PNAS & 308 & 5 & PNAS & 5916 \\
\hline 6 & Nature Communications & 205 & 6 & Science (AAAS) News & 5914 \\
\hline 7 & PLoS ONE & 165 & 7 & British Medical Journal (Clinical Research Edition) & 5561 \\
\hline 8 & Cell Reports & 160 & 8 & Scientific Reports & 4716 \\
\hline 9 & New England Journal of Medicine & 156 & 9 & Science & 4133 \\
\hline 10 & SSRN & 152 & 10 & Angewandte Chemie.International Edition & 3809 \\
\hline 11 & Molecular Cell & 113 & 11 & The Lancet & 3342 \\
\hline 12 & Journal of Clinical Oncology & 112 & 12 & Nature Communications & 3101 \\
\hline 13 & Science Translational Medicine & 107 & 13 & Figshare & 2998 \\
\hline 14 & Neuron & 99 & 14 & JAMA & 2939 \\
\hline 15 & The Lancet & 99 & 15 & Physical Review Letters & 2844 \\
\hline
\end{tabular}

*Shared top posted sources are denoted in bold.

mentioned by 10,606 Weibo posts, while 37,986 sources were mentioned by 3.16 million tweets. The number of both the sources and posts of Weibo were dwarfed by those of Twitter.

Table 10 lists the top 15 weiboed and tweeted sources respectively. From Table 10, Weibo shares many top posted sources with Twitter. In addition to traditional multidisciplinary journals like Nature, Science, PNAS and prestigious professional journals like The Lancet, open access journals and preprint platforms like PLoS ONE and arXiv are also widely discussed on Weibo and Twitter. Meanwhile, Weibo has its own emphasis on some specific journals which reflects Chinese local attention. For example, Cell, along with Science and Nature (SNC), are all considered highly recognized top-tier journals in China (Shao \& 


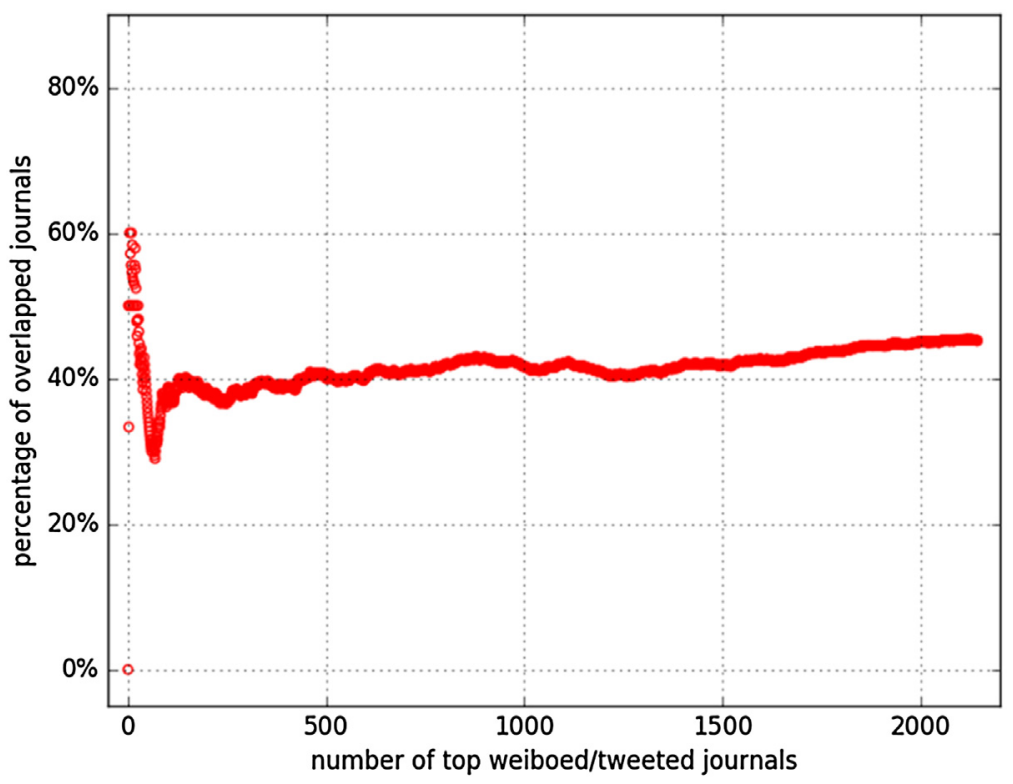

Fig. 7. Overlapped journals of weiboed \& tweeted journals.

Shen, 2011). According to Table 10, Cell gains high attention on Weibo, but not equal attention on Twitter, being ranked 87th among all the highly tweeted journals. Other examples are Science Translational Medicine and Neuron, which are both highly mentioned on Weibo but much less visible on Twitter. In general, multidisciplinary journals and medical science sources get the most attention on Weibo. Twitter users pay much attention to chemical science sources, for example, Angewandte Chemie International Edition (a German chemical journal) and JAMA.

In addition, a Spearman's rank-order correlation was run to determine the relationship between the lists of weiboed and tweeted sources. In total, 2414 sources are mentioned on Weibo; 37986 sources are mentioned on Twitter. 2006 sources are mentioned by both Weibo and Twitter. There is a strong, positive correlation between these two list of sources $\left(r_{s}(2006)=0.75, p=0.00\right)$.

As observed above, journals that are both highly weiboed and tweeted have high Impact Factors. To explore the relationship between Impact Factor and the attention on both Weibo and Twitter, we first examined how much common journals Weibo and Twitter users pay attention to. Fig. 7 shows that largely, around $40 \%$ of journals are both weiboed and tweeted, regardless of the number of top journals we look at. Particularly, the most top weiboed and tweeted articles overlap even more. We then examined whether weiboed or tweeted journals have higher Impact Factors. The average JIF (Journal Impact Factor) percentile reported in JCR (Journal Citation Reports) 2015, designed for measuring Impact Factor of journals across field, is used for comparison's purpose. Fig. 8 shows that the average JIF percentiles of the tweeted journals are evenly distributed, with approximately $5 \%$ of the tweeted journals falling on each level of average JIF percentile. Compared with Twitter, weiboed journals have their average JIF percentiles more prevailingly falling on the higher levels. For example, more than $15 \%$ of weiboed journals have average JIF percentiles higher than $95 \%$. This indicates that Weibo users pay more attention to higher impact journals, but Twitter users do not have a preference on high impact journals.

\subsection{Distribution of Weibo altmetrics on the discipline level}

There are significant disciplinary differences regarding citation indicators. Comparison between citations of physics publications and that of psychology ones, for example, can thus be misleading. We investigate the disciplinary differences of Weibo altmetrics based on $S_{s}$ (see Methodology, Section 3.6). From Fig. 9, we see that the most frequently weiboed articles are from General disciplines (20.5\%). This means that interdisciplinary content gains the most attention from Weibo. Biochemistry, Genetics and Molecular Biology (14.4\%) is ranked the second. It's followed by Health sciences (13.4\%) and Medicine (13.2\%), which get the third and fourth position respectively. The next is "Life Sciences", which is ranked the fifth. These five disciplines are the most frequently weiboed disciplines. Each of them has a percentage over $10 \%$, and they together occupy $72.3 \%$ of all the weiboed articles. The following disciplines are "Social Sciences" (6.4\%), "Physical Sciences" (4.2\%), "Neuroscience" (2.2\%), "Psychology" (1.8\%), "Economics, Econometrics and Finance" (1.7\%), "Agricultural and Biological Sciences" (1.5\%), and "Physics and Astronomy" (1.2\%). These 7 disciplines have percentages over $1 \%$ and together occupy $19.1 \%$ of all weiboed articles. The rest 19 disciplines occupy the remaining $8.6 \%$ of all the weiboed articles.

Twitter users, on the other hand, discuss mostly about Health Science discipline (22.1\%), and then Medicine (20.8\%) and Life Science (10.7\%), followed by Social Science (7.6\%) and Physical Science (7\%). The fact that both Weibo and Twitter users 


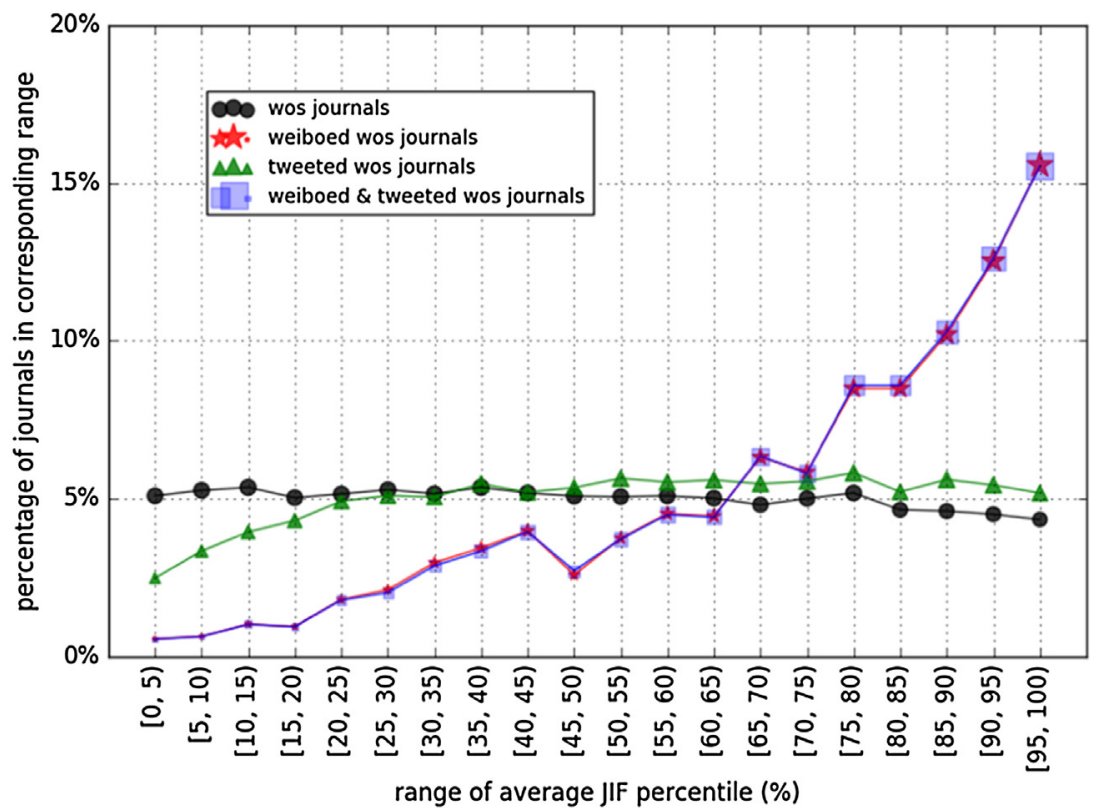

Fig. 8. Relationship between Impact Factor and attention from Weibo/Twitter.

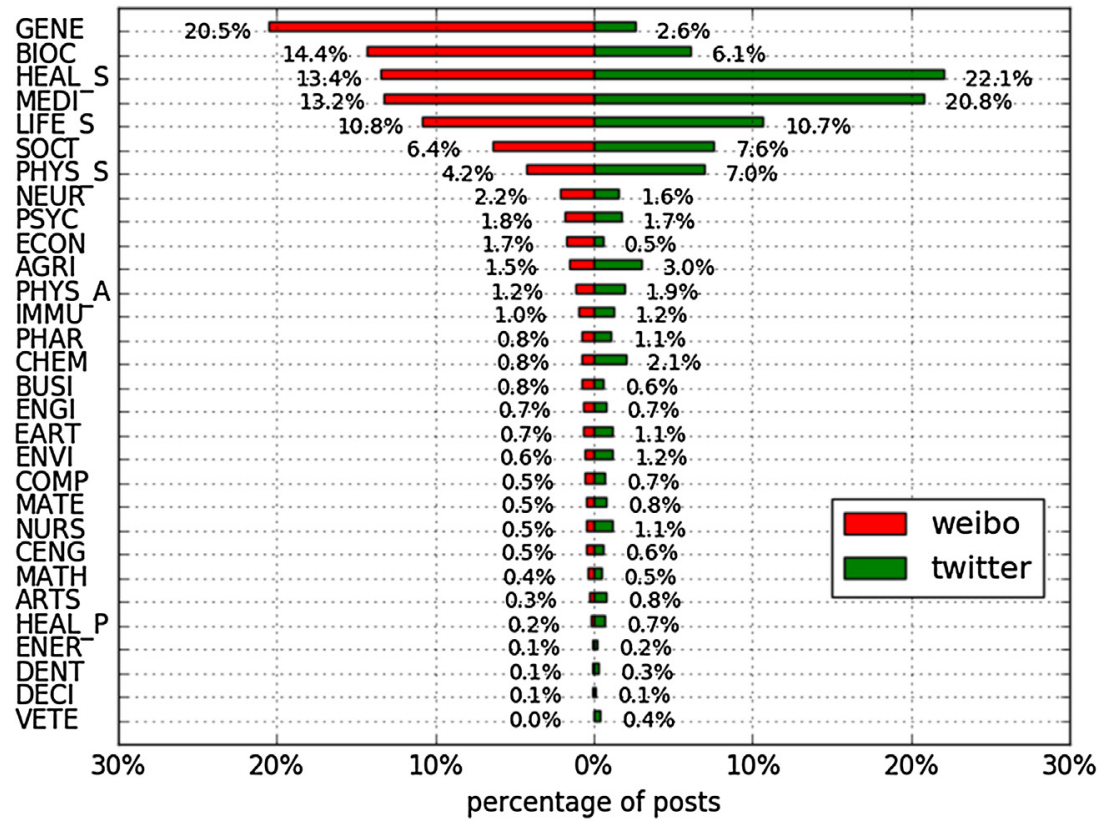

Fig. 9. Discipline distribution of Weibo altmetrics (based on $\boldsymbol{S}_{\boldsymbol{s}}$ )..

discuss extensively about Health Science and Medicine indicates that these two disciplines are of special interest to users of microblog services. Meanwhile, Weibo users pay the most attention to General and Biochemistry, Genetics and Molecular Biology, showing Chinese scientists' attention to these two disciplines.

While the discipline distribution based on $S_{s}$ provides a macro view, the distribution based on $S_{p}$ and $S_{\mathrm{m}}$ provide further insight as shown in Table 11. From Table 11, we see discipline distributions based on $S_{p}$ and $S_{m}$ are basically in accordance with that based on $\mathrm{S}_{s}$, but with more details. For example, under general "Biology Sciences", it is found that "Cell biology" (8.6\%, $\left.\boldsymbol{S}_{\boldsymbol{m}} \# 3\right)$, "Molecular biology" (3.3\%, $\left.\boldsymbol{S}_{\boldsymbol{m}} \# 6\right)$, and "Genetics" $\left(1.1 \%, \boldsymbol{S}_{\boldsymbol{m}} \# 18\right)$ are more mentioned and discussed by Weibo users. Under "Medical Sciences", it is found that "Clinical Sciences" (4\%, $\left.S_{p} \# 5\right)$, "Oncology" (3.3\%, $\left.\boldsymbol{S}_{\mathrm{p}} \# 7 ; 5.3 \%, \boldsymbol{S}_{\boldsymbol{m}} \# 4\right)$, "Neurosciences" (3.4\%, $\left.\boldsymbol{S}_{\mathrm{p}} \# 6 ; 4.4 \%, \boldsymbol{S}_{\boldsymbol{m}} \# 5\right)$, and "Allergy and immunology" $\left(2 \%, \boldsymbol{S}_{\boldsymbol{m}} \# 9\right)$ obtain relatively higher Weibo attention. Under the 


\begin{tabular}{llll}
\hline $\mathbf{N}$ & $\boldsymbol{S}_{\boldsymbol{p}}$ & $\mathbf{P .}$ & $\boldsymbol{S}_{\boldsymbol{m}}$ \\
\hline 1 & Medical and Health Sciences & $19.8 \%$ & Pcience \\
2 & Biological Sciences & $11.5 \%$ & Medicine \\
3 & Multidisciplinary & $9.2 \%$ & Cell biology \\
4 & Cognitive Science & $4.3 \%$ & Neoplasms \\
5 & Clinical Sciences & $4.0 \%$ & Neurology \\
6 & Neurosciences & $3.6 \%$ & Molecular biology \\
7 & Oncology and Carcinogenesis & $3.4 \%$ & Biology \\
8 & Psychology & $3.3 \%$ & $3.3 \%$ \\
9 & Psychology and Cognitive Sciences & $3.1 \%$ & Psychology \\
10 & Public Health and Health Services & $3.0 \%$ & Allergy and immunology \\
11 & Biochemistry and Cell Biology & $2.0 \%$ & Biotechnology \\
12 & Chemical Sciences & $1.8 \%$ & Nutritional sciences \\
13 & Information and Computing Sciences & $1.7 \%$ & Chemistry \\
14 & Immunology & $1.5 \%$ & Clinical laboratory techniques \\
15 & Physical Sciences & $1.2 \%$ & Genetics medical \\
16 & Engineering & $1.2 \%$ & Endocrinology \\
17 & Economics & $1.2 \%$ & $1.6 \%$ \\
18 & Political Science & $1.0 \%$ & Pediatrics \\
& & $1.0 \%$ & Nanotechnology \\
\hline
\end{tabular}

${ }^{*} \boldsymbol{S}_{\mathrm{p}} \# 1$ represents No.1 in $\boldsymbol{S}_{\mathrm{p}}$ category.

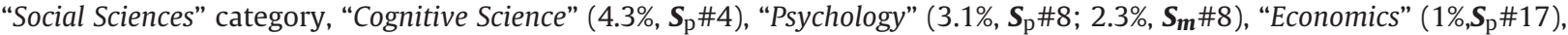
and "Political Science" $\left(\boldsymbol{S}_{\mathrm{p}} \# 18\right)$ are most frequently weiboed.

\section{Discussion}

This is a type-B study, which pays particular attention to how international research is discussed locally. The paper shows that the relative coverage of Weibo altmetrics over global science is $0.5 \%$. This percentage is similar to Peer Review (0.5\%), and higher than several global altmetrics sources like Video (0.4\%) and Q\&A (0.2\%). However, it is significantly dwarfed by Twitter (65.7\%). This shows different levels of attention between a local and a global microblog service. By combining our finding with the previously measured absolute coverage, for example, the Twitter coverage of PubMed articles (Haustein et al., 2014c), the absolute coverage of Weibo can be estimated. Chinese scholars have been calling on establishing Chinese altmetrics infrastructure. The full scholarly communication activeness of Weibo on both domestic and international research should be measured in the future.

Immediacy can be a relative concept according to different interpretations and research goals. Compared with the citation window, captures within 180 days on Weibo are acceptable to be defined as immediate. The study shows proof of the immediacy of Weibo, Twitter and general altmetrics. $69 \%$ of weiboed articles received their first weibo within 180 days of publication, $44 \%$ were within 7 days, and $7 \%$ were prior to formal publishing. In contrast, $64 \%$ of tweeted articles had their first tweet within 180 days.

The distribution of Weibo altmetrics is, compared with citations, more skewed on the article level, is more concentrated on the source level, and presents significant differences on the discipline level. Specifically, $20 \%$ of weiboed articles are mentioned by $70 \%$ of the weibos. The average number of weibos each article receives is 3.5 , despite the fact that $84 \%$ of the weiboed articles receive no more than 3 weibos. Twitter has this similar pattern $-20 \%$ of tweeted articles obtains $77 \%$ of tweets. Each article receives an average of 6.2 articles, and 74\% of the tweeted articles had no more than 3 tweets. The high skewness indicates that Weibo altmetrics is able to distinguish featured studies. The source distribution of Weibo altmetrics is concentrated, even more so than Bradford's law distribution. This reflects China's biased attention to prestigious journals, because Chinese universities and institutions have unbalanced rewards on publications in these journals (Shao \& Shen, 2012; Hvistendahl, 2013). In contrast, Twitter users discuss more diverse sources, reflected in that Figshare is also among the top tweeted sources. Nevertheless, both Weibo and Twitter users pay much attention to medical sources, which indicates that medical research is of similar level of interest to China and other nations.

Content analysis of weibos discussing highly weiboed articles is especially useful in explaining the value of the scientific weibos. It is found that to disseminate by giving perfunctory introduction was the most common motivation for posting the scientific weibos. Moreover, to highlight or elaborate the academic usage, practical usage, interesting parts or surprising parts are all common motivations for weiboing an article. This is in accordance with a previous study that shows highly tweeted articles tend to be curious or funny, have potential health applications, and concern the whole scholarly community (Haustein et al., 2014c). Another previous content analysis (Thelwal, Tsou, Weingart, Holmberg, \& Haustein, 2014) finds that 42\% of scientific tweets echo the article title. Our study showed, however, that conclusion (23\%) is the most frequently mentioned element of articles on Weibo, and $33 \%$ of the weibos do not mention the article at all. 


\section{Conclusion}

The study, based on a large-scale dataset, reveals characteristics of Sina Weibo as an important local altmetrics platform on which global science is discussed. We conducted a systematic comparison between Weibo and the global microblogging platform, Twitter, aiming to draw attention to local altmetrics in developing countries, to investigate the common features of microblog altmetrics, and to shed light on cultural differences in scholarly communication on these social media platforms.

Sina Weibo, as a local social media platform in China, is meanwhile the world's second largest microblog service. Weibo users discuss global science more actively than it is discussed on several global altmetrics sources. Being a channel connecting global science and regional community, local social media should be considered as an integral part of the altmetrics landscape. Strong evidence shows that Weibo, as well as Twitter and general altmetrics, have significant immediacy advantage over citations, endorsing earlier claims that altmetrics can potentially detect more realtime impact of research products.

The highly skewed distribution of Weibo and Twitter altmetrics is not surprising considering their nature of social media (Banditwattanawong, Masdisornchote, \& Uthayopas, 2014; Jiang, Wang, Yang, \& Li, 2015). However, certain types of articles, a number of journals and specific disciplines are found to attract more Weibo attention. The common motivations for posting scientific weibos is to disseminate or discuss the articles because they are interesting, surprising, academically useful, or practically useful. Weibo users pay particular attention on prestigious journals because of the Chinese academic reward system, which has biased awards for publications in these journals. In addition, discussion of articles published on important open access platforms confirms China's acceptance of new forms of scientific communication. While focusing the most on General and "Biochemistry, Genetics and Molecular Biology", Weibo users share interest in top discussed disciplines such as Health science, Medicine and Life Science, with Twitter. These disciplines with comparatively higher coverage of altmetrics, above other disciplines, can make use of social media scholarly communication and altmetrics to better achieve their development goals.

This study considers only articles. However, other types of research products also matter. As altmetrics studies evolve, researchers have noted that Twitter accounts are of different backgrounds, for example, automatic bots maintained by individuals or institutions (Haustein et al., 2016). Different user categories are also shown to have influence on the value of tweets (Yu, 2016b). Therefore, more content analysis taking context information into account is needed, to further reveal the nature of Weibo and general altmetrics.

\section{Acknowledgements}

This research was supported by China Scholarship Council (NO. 201506270024) and sponsored by Humanities and Social Science Foundation by the Ministry of Education of China (16YJA870011) and the Research Center of Information Technology \& Economic and Social Development in Zhejiang Province. The authors thank Altmetric.com for providing the dataset and Professor Junping Qiu for his insightful comments.

\section{Appendix A.}

See Table A1.

\section{Table A1}

Abbreviation of Scopus disciplines

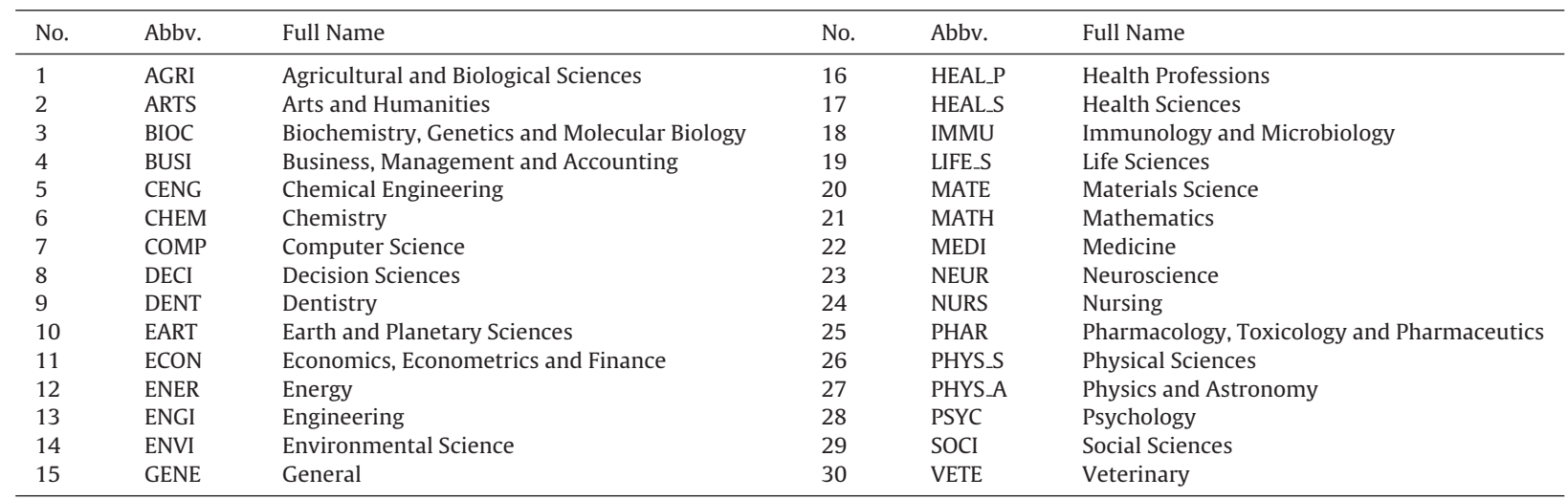

\section{References}


Alperin, J. P. (2015). Geographic variation in social media metrics: an analysis of Latin American journal articles. Aslib Journal of Information Management, 67(3), 289-304.

Altmetric LLP. (2016). How is the Altmetric Attention Saltmetric attention score calculated? https://goo.gl/nDKbCL

Araújo, R. F., Murakami, T. R. M., Lara, J. L. D., \& Fausto, S. (2015). Does the Global South have altmetrics? analyzing a Brazilian LIS journal. Proceedings of ISSI 2015-15th international conference of the international society for scientometrics and informetrics, 111-112.

Banditwattanawong, T., Masdisornchote, M., \& Uthayopas, P. (2014). The smart distribution of social media contents. In Electrical engineering congress (iEECON) 2014 international (pp. 1-4).

Brody, T., \& Harnad, S. (2006). Earlier web usage statistics as predictors of later citation impact. Journal of the American Society for Information Science and Technology, 57(8), 1060-1072.

Das, T. (2015). Measuring scholarly use of government information: An altmetrics analysis of federal statistics. Government Information Quarterly, 32(3), $246-252$.

Friedrich, N., Bowman, T. D., Stock, W. G., \& Haustein, S. (2015). Adapting sentiment analysis for tweets linking to scientific papers. https://arxiv.org/abs/1507.01967

Garfield, E. (1972). Citation analysis as a tool in journal evaluation. Science, 1972(178), 471-479.

Garfield, E. (1998). Random thoughts on citationology its theory and practice. Scientometrics, 43(1), 69-76.

Haustein, S., Bowman, T. D., Macaluso, B., Sugimoto, C. R., \& Larivière, V. (2014). Measuring Twitter activity of arXiv e-prints and published papers. https://dx.doi.org/10.6084/m9.figshare.1041514.v1

Haustein, S., Peters, I., Bar-Ilan, J., Priem, J., Shema, H., \& Terliesner, J. (2014). Coverage and adoption of altmetrics sources in the bibliometric community. Scientometrics, 101(2), 1145-1163.

Haustein, S., Peters, I., Sugimoto, C. R., Thelwall, M., \& Larivière, V. (2014). Tweeting biomedicine: An analysis of tweets and citations in the biomedical literature. Journal of the Association for Information Science and Technology, 65(4), 656-669.

Haustein, S., Bowman, T. D., \& Costas, R. (2015). When is an article actually published? An analysis of online availability, publication, and indexation dates. https://arxiv.org/abs/1505.00796

Haustein, S., Bowman, T. D., Holmberg, K., Tsou, A., Sugimoto, C. R., \& Larivière, V. (2016). Tweets as impact indicators: examining the implications of automated bot accounts on Twitter. Journal of the Association for Information Science and Technology, 67(1), 232-238.

Holmberg, K., \& Thelwall, M. (2014). Disciplinary differences in Twitter scholarly communication. Scientometrics, 101(2), $1027-1042$.

Hvistendahl, M. (2013). China's publication bazaar. Science, 342(6162), 1035-1039.

ImpactStory. (2016). Let's value the software that powers science: Introducing depsy. http://blog.impactstory.org/introducing-depsy

Jiang, B., Wang, L., Yang, C., \& Li, R. (2015). Mining multiplex power-law distributions and retweeting patterns on Twitter. Fuzzy systems and knowledge discovery (FSKD), 2015 12th international conference, 1437-1443.

Kolahi, J. (2015). Altmetrics: A new emerging issue for dental research scientists. Dental Hypotheses, 6(1), 1-5.

Kousha, K., Thelwall, M., \& Abdoli, M. (2012). The role of online videos in research communication: A content analysis of YouTube videos cited in academic publications. Journal of the American Society for Information Science and Technology, 63(9), 1710-1727.

Kraker, P., Lex, E., Gorraiz, J., Gumpenberger, C., \& Peters, I. (2015). Research data explored II: The anatomy and reception of Figshare. http://arxiv.org/abs/1503.01298

Liu, J., Konkiel, S., \& Williams, C. (2015). Understanding the impact of research on policy using Altmetric data. [Retrieved December 29, 2016, from http://figshare.com/articles/Understanding_the_impact_of_research_on_policy_using_Altmetric_data/1439723]

Liu, B. (2012). Grass-root popular science in the context of new medium era: An amateur author's understandings and experience of popular science writing. Science Popularization, 6, 4-9.

Loach, T. V., \& Evans, T. S. (2015). Ranking journals using altmetrics. http://arxiv.org/abs/1507.00451

Moed, H. F. (2015). Altmetrics as traces of the computerization of the research process. https://arxiv.org/abs/1510.05131

Mohammadi, E., \& Thelwall, M. (2013). Assessing non-standard article impact using F1000 labels. Scientometrics, 97(2), $383-395$.

Na, J. C. (2015). User motivations for tweeting research articles: A content analysis approach. Proceedings of the 17th international conference on asian digital libraries, 197-208.

Peters, I., Jobmann, A., Eppelin, A., Hoffmann, C. P., Künne, S., \& Wollnik-Korn, G. (2014). Altmetrics for large, multidisciplinary research groups: comparison of current tools. Bibliometrie- Praxis Und Forschung, 3(1), 1-19.

Peters, I., Kraker, P., Lex, E., Gumpenberger, C., \& Gorraiz, J. (2015). Research data explored: Citations versus altmetrics. https://arxiv.org/abs/1501.03342

Poplašen, L., \& Zrnić, L. (2014). Altmetrics -new metrics and its application in Croatia. In Libraries in the digital age (LIDA) proceedings

http://ozk.unizd.hr/proceedings/index.php/lida/article/view/132

Priem, J., Taraborelli, D., Groth, P., \& Neylon, C. (2010). Altmetrics: A manifesto. http://altmetrics.org/manifesto/

Priem, J. (2013). Scholarship: Beyond the paper? Nature, 495(7442), 437-440.

Qiu, J. P., \& Yu, H. Q. (2015). Some basic problems in advancing the development of Altmetrics. The Journal of Library Science in China, 41(1), 4-15, in Chinese.

Rehemtula, S., Rosa, M., Leitão, P., \& Avilés, R. (2014). Altmetrics in institutional repositories: new perspectives for assessing research impact. In Libraries in the digital age (LIDA) proceedings http://ozk.unizd.hr/proceedings/index.php/lida/article/view/141

ScienceOpen GmbH. http://goo.gl/GzTP5o

Seglen, P. O. (1992). The skewness of science. Journal of the American Society for Information Science, 43(9), 628.

Shao, J. F., \& Shen, H. Y. (2011). The outflow of academic papers from China: why is it happening and can it be stemmed? Learned Publishing, $24(2), 95-97$.

Shao, J. F., \& Shen, H. Y. (2012). Research assessment and monetary rewards: the overemphasized impact factor in China. Research Evaluation, 21(3), 199-203.

Shema, H., Bar-Ilan, J., \& Thelwall, M. (2012). Research blogs and the discussion of scholarly information. PLoS One, 7(5), e35869.

Shema, H., Bar-Ilan, J., \& Thelwall, M. (2014). Do blog citations correlate with a higher number of future citations? Research blogs as a potential source for alternative metrics. Journal of the Association for Information Science and Technology, 65, 1018-1027.

Tammaro, A. M. (2014). Altmetrics in the humanities: Perceptions of Italian scholars. In Libraries in the digital age (LIDA) proceedings http://ozk.unizd.hr/proceedings/index.php/lida/article/view/167

Thelwall, M., \& Kousha, K. (2015). ResearchGate: Disseminating, communicating, and measuring scholarship? Journal of the Association for Information Science and Technology, 66(5), 876-889.

Thelwall, M., \& Wilson, P. (2015). Mendeley readership altmetrics for medical articles: an analysis of 45 fields. Journal of the Association for Information Science and Technology, http://dx.doi.org/10.1002/asi.23501

Thelwall, M., Tsou, A., Weingart, S., Holmberg, K., \& Haustein, S. (2013). Tweeting links to academic articles. http://citeseerx.ist.psu.edu/viewdoc/download?doi=10.1.1.385.6234\&rep=rep1\&type=pdf

Torres, D., Cabezas, A., \& Jiménez, E. (2013). Altmetrics: New indicators for scientific communication in web 2.0. http://arxiv.org/abs/1306.6595

Vickery, B. C. (1948). Bradford's law of scattering. Journal of Documentation, 4(3), 198-203.

Wang, Y., Guo, S., \& Zhang, J. (2015). Study on correlation of researcher influence indicators. Library and Information Service, 5(5), 29-38.

Yu, H. Q. (2016a). Context of Altmetrics data matters: An investigation of count type and user category. Scientometrics, http://dx.doi.org/10.1007/s11192-017-2251-z

Yu, H. Q. (2016b). Large scale disciplinary correlation analysis between altmetrics indicators and citations. Doctoral Forum of Chinese Information Science, 12-21. 
Zahedi, Z., Costas, R., \& Wouters, P. (2014). How well developed are altmetrics? A cross-disciplinary analysis of the presence of 'alternative metrics' in scientific publications. Scientometrics, 101(2), 1491-1513.

Zahedi, Z., Fenner, M., \& Costas, R. (2014). How consistent are altmetrics providers? Study of 1000 PLOS ONE publications using the PLOS ALM, Mendeley and Amendeley altmetric.com APIs. https://dx.doi.org/10.6084/m9.figshare.1041821. v2

Zhao, F., Chen, Y., Ge, S., Yu, X., \& Shao, S. (2014). A quantitative analysis of the mass media coverage of genomics medicine in china: A call for science journalism in the developing world. Omics: A Journal of Integrative Biology, 18(4), 222-230. 